Children's biological givens, stress responses, language and cognitive abilities and family background after entering kindergarten in toddlerhood

\title{
Suhonen, Eira
}

2018

Suhonen , E , Sajaniemi , N K , Alijoki , A \& Nislin , M A 2018 , ' Children's biological givens, stress responses, language and cognitive abilities and family background after entering kindergarten in toddlerhood ', Early Child Development and Care, vol. 188 , no. 3 , pp. 345-358 . https://doi.org/10.1080/03004430.2016.1218157

http://hdl.handle.net/10138/325385

https://doi.org/10.1080/03004430.2016.1218157

unspecified

acceptedVersion

Downloaded from Helda, University of Helsinki institutional repository.

This is an electronic reprint of the original article.

This reprint may differ from the original in pagination and typographic detail.

Please cite the original version. 
Children's biological givens, stress responses, language and cognitive abilities, and family background after entering kindergarten in toddlerhood

Corresponding author:

Eira Suhonen, PhD., University lecturer, Special teacher

University of Helsinki

Faculty of Behavioral Sciences

Department of Teacher Education

Special Education Section

P.O. Box 8, Siltavuorenpenger 10 A FI-00014 University of Helsinki, Finland

eira.suhonen@helsinki.fi

$+358445523035$

Eira Suhonen is a university lecturer at the University of Helsinki. Her main research interest is in early childhood special education. She has focused in her research on play, early intervention and children with special educational needs.

Nina K Sajaniemi, PhD, Adjunct Professor

University of Helsinki

Faculty of Behavioral Sciences

Department of Teacher Education

Early Education Section

P.O. Box 8, Siltavuorenpenger 10 FI-00014 University of Helsinki, Finland

nina.sajaniemi@helsinki.fi

$+358503182986$ 
Nina Sajaniemi is adjunct professor, principal investigator and head of early childhood education (academic affairs) in University of Helsinki. She is specialized in developmental neuropsychology, interpersonal neurobiology and stress regulation.

Alisa Alijoki, $\mathrm{PhD}$, University lecturer

University of Helsinki

Faculty of Behavioral Sciences

Department of Teacher Education

Early Education Section

P.O. Box 8, Siltavuorenpenger 10 FI-00014 University of Helsinki, Finland

alisa.alijoki@helsinki.fi

$+358504125589$

Alisa Alijoki is a university lecturer at the University of Helsinki. She is specialized in early intervention and transitions from kindergarten to school.

Mari A Nislin, PhD., Special teacher

University of Helsinki

Faculty of Behavioral Sciences

Department of Teacher Education

Special Education Section

P.O. Box 8, Siltavuorenpenger 10 A FI-00014 University of Helsinki, Finland

mari.nislin@helsinki.fi

$+358405911559$

Mari Nislin is a post-doctoral researcher at the University of Helsinki. Her research interests are in stress response regulation and occupational well-being among early childhood professionals. In addition, she is investigating professionalism in early childhood education. She is specialised in analyses of biomarkers of stress response regulation both in adults and children. 
This work was supported by the [Academy of Finland] under Grant [264425].

\begin{abstract}
We aimed to investigate stress response regulation, temperament, cognitive and language abilities and family socioeconomic status (SES) in children who entered kindergarten before two years of age. Whilst children's stress regulatory systems are vulnerable to environmental influences, little is known about how temperament and family characteristics impact stress regulation in a child's early years of development. The study participants were 129 children between the ages of 7 years and 23 months from 29 kindergartens. Stress response regulation was assessed by measuring salivary cortisol and alpha-amylase activity. Cognitive and language abilities were assessed using Bayley-III and the children's temperaments were assessed using the Early Childhood Behavior Questionnaire (ECBQ). Family characteristics (SES, parental stress) were assessed using surveys. The results suggest that children are alert during their kindergarten day, but their stress response regulation is balanced. Girls and boys differed in cognitive and language abilities, but variation in language had a greater impact on learning difficulties in a family than it did on gender. We propose that children's individual needs should be better acknowledged in early childhood education.
\end{abstract}

Keywords: Stress response regulation, early childhood education, temperament, cognitive and language abilities, salivary cortisol and alpha-amylase, family SES

\title{
1. Introduction
}

Children attend kindergarten with various early developmental histories. Each child is born with a unique temperament and other biological givens. In addition, socioeconomic environment, parental attitudes and parenting styles impact the developmental trajectories of children, even in utero. It is widely known that an adverse environment and poor parenting can have cumulative jeopardizing effects on a child's development, whereas a loving, caring environment that provides steady socioeconomic growth protects that development (Sourander, 2015).

A child's early years form the fundamental basis for future learning and wellbeing. The first three years of life are a period of incredible growth in all areas of human development. Brains are extremely plastic in early life, which can have positive and negative consequences. On the positive side, young children's brains are open to learning and enriching influences. On the negative side, young children's brains are vulnerable to the effects of an impoverished or un-nurturing environment (identifying author, 2015).

Environmental influences, especially early in life, have been convincingly shown to have an effect on social, emotional and cognitive development in childhood (Rutter et al., 2015). A primary pathway through which the environment exerts this influence is via changes in stress-responsive biological systems, especially the sympathetic and parasympathetic branches of the autonomic nervous system (ANS), the hypothalamic-pituitary-adrenal (HPA) axis and their target tissues, including the brain (Berntson, Cacioppo, \& Quigley, 1993; Cicchetti \& Rogosch, 2012; Porges, 2007; Sapolsky, Romero, \& Munck, 2000). 
Stress-induced neurobiological responses guide adaptive and essential responses to environmental changes and challenges. Activation of the stress reactive system is requisite for becoming alert and attentive (Sajaniemi et al., 2015; Siegel, 2005). Regulation of stress responses is mandatory for maintaining balanced activity when the brain and body are preparing to take action and when it is needed to delay immediate reactivity in striving to achieve current or future goals. Thus, stress response activation might be the initial stage of learning processes. Without regulation, stress activation results in energy-consuming fight, flight or freeze responses. Recurring immediate reactivity restrains neural activation in the prefrontal cortex and endangers healthy development in young maturing brain (identifying author, 2015; Gunnar \& Cheatham, 2003).

An impressive body of research has accurately outlined the processes underlying these stress responses (De Kloet, Fitzsimons, Datson, Meijer, \& Vreugdenhil, 2009; Gunnar, Wewerka, Frenn, Long \& Griggs, 2009; Porges, 2007). Autonomic arousal is associated with acute, short-term responses from the Sympatho-Adrenal-Medullary (SAM) axis and more chronic, longer-term responses from the Hypothalamus-Pituitary-Adrenal (HPA) axis. Even though the SAM axis and the HPA axis systems function in a coordinated manner to regulate stress responses (see Sapolsky et al., 2000; Granger, Kivlighan, El-Sheikh, Gordis, \& Stroud, 2007), it has been proposed that the relationship between the HPA and SAM systems is asymmetric and reciprocal, with opposite patterns of diurnal change evidenced in decreasing cortisol and increasing salivary alpha-amylase (sAA) throughout the day (Nater et al., 2007). In addition, the increase and decrease in sAA in response to stress precede those in cortisol, leading to higher cortisol but lower sAA approximately 10 minutes after the stressor has triggered it (Gordis, Granger, Susman, \& Trickett, 2006; Engert et al., 2011).

The diurnal rhythm of cortisol in children is still under progression. Infants are born without a diurnal cortisol rhythm; it emerges during the first 18 months of life. It has even been suggested that the maturation of the HPA axis is ongoing up to the third year of life (Watamura, Donzella, Kertes, \& Gunnar, 2004). In addition, baseline cortisol levels seem to decrease across infancy and early childhood (Blair et al., 2011). Less is known about the maturation of the sAA axis. It has been documented that basal sAA levels increase between 2 months and 24 months of age (Davis \& Granger, 2009). However, evidence about the maturation of the diurnal sAA rhythm is almost nonexistent.

Children with particular temperaments might be more vulnerable to early life experiences, and some individual differences might lead to greater sensitivity to environmental variations (Boyce \& Ellis, 2005). Sensitivity to the environment encompasses both individual differences in physiological reactivity to stress and individual temperament differences that are highly associated with stress responses. Reactive temperament is one of those individual difference factors that have been hypothesised to enhance sensitivity to both positive and negative qualities of the environment during early childhood development (Belsky, 2005; Obradovic \& Boyce, 2009). Behaviourally inhibited, reactive children display a heightened orientation to threat, enhanced novelty detection and greater error monitoring; they are also more likely to develop anxiety disorders (Fox \& ReedSutrerland, 2010).

Studies have proposed that affectivity has an effect on stress regulation as well. Low positive affectivity, concurrent elevated morning cortisol and cortisol dysregulation have been observed in young children (Dougherty, Klain, Olino, Dyson, \& Rose, 2009). Furthermore, children who have exhibited higher negative affectivity at the age of three have evidenced elevated evening cortisol at the age of six (Dougherty et al., 2013). Children with high negative affectivity or low positive 
affectivity may have greater difficulty modulating their biological and behavioural stress responses, making them more susceptible to the cumulative challenges of the day and, increasing, the risk for later depression (Dougherty et al., 2013). In addition, some studies have revealed associations with externalising or internalising behaviours and low or high baseline cortisol, respectively (HillSoderlund et al., 2015; Shirtcliff, Granger, Booth, \& Johnson, 2005; van Goozen, Matthys, CohenKettenis, Buitelar, \& van Engeland, 2000; McBurnett, Lahey, Rathou, \& Loeber, 2000; Gunnar, Tout, de Haan, Pierce, \& Stanbury, 1997; Hart \& Risley, 1995).

Fewer studies have examined the relationship between sAA and emotional or behavioural problems. However, high externalising behaviour has been documented in children with either high or low levels of sAA (Keller \& El-Sheikh, 2009). The link between high levels of sAA, approach tendency and positive emotion, as well as between high levels of cortisol, withdrawal tendency and negative emotion, has also been documented (Fortunato, Dridin, Stranger, \& Buss, 2008). ElSheikh, Erath, Vuckhalt, Granger, and Mize (2008) reported that higher resting cortisol concentrations were associated with higher levels of internalising and externalising problems, but only in conjunction with high sAA concentrations. Behavioural problems were most apparent in children with both high cortisol and high sAA levels. In contrast, children with high sAA but low cortisol levels seemed to have the least number of behaviour problems. Similar moderated relationships have been noted between cortisol and sAA reactivity during laboratory-based psychological stress paradigms (Gordis et al., 2006), although the crossover nature of the interactions have been somewhat inconsistent across studies (Allwood, Handwerger, Kivlighan, Granger, \& Stroud, 2011).

It has been suggested that the relationship between cognitive development and stress regulation evolves over time (Hackmann \& Farah 2009). Some studies have found that low cognitive performance in school-aged children precedes the flat diurnal cortisol pattern and low morning cortisol levels seen in mid-adulthood (Power, Li, \& Hertzman, 2008). In addition, Evans et al. (2011) reported an inverse relationship between age and cognitive performance with an attenuated cortisol awakening response. Increases in cortisol responses to a challenge have been shown to be related to better cognitive performance in children (Blair, Granger, \& Petersrazza, 2005). Optimal cognitive outcomes have been shown to be associated with asymmetrical cortisol/sAA profiles (Keller et al., 2012). Keller et al. (2012) found that higher cortisol levels were predictive of lower cognitive abilities in toddlers with moderate to high levels of sAA. However, higher sAA levels were predictive of better cognitive abilities in toddlers with concurrently moderate to low levels of cortisol (Berry et al., 2012). Furthermore, diurnal cortisol patterns characterised by a more positive awakening response and lower cortisol levels during the day, which indicates higher cortisol reactivity and lower stress levels, were associated with better language and cognitive scores early in toddlerhood (Saridjan et al., 2014). In toddlerhood, the structure of cognitive development is not yet clear and the emergence of the processes of language and non-verbal language are distinct. Studies focusing on both language abilities and non-verbal cognitive abilities in relation to stress response regulation are rare. Our study is among the first to examine diurnal cortisol and concurrent diurnal sAA levels in relation to language and cognitive performance in young children.

Maturation of stress response regulation in children might be linked to socioeconomic status (SES). Karb, Elliot, Dowd, and Morenoff (2012) documented that a stressful environment with economic pressures, living in an unprivileged neighbourhood and a low level of social support are linked with blunted cortisol patterns in adults, indicating chronic stress throughout the day. Studies have documented that children growing up in low SES conditions experience more disorganised and chaotic environments with a greater level of noise and unpredictably (Evans, 2004; Vernon-Feagans et al., 2012). Some studies have found that these types of environments result in higher levels of 
basal cortisol (see Blair et al., 2011). Low SES has been associated with increased diurnal levels of cortisol among infants (Saridjan et al., 2010) and higher basal levels among preschool aged children (Blair et al., 2005). However, some studies have found either no associations or mixed associations between SES and cortisol (Down, Simanek, \& Aiello, 2009). There is less data (and mixed data) on the relationship between sAA and SES. Children from low SES backgrounds have been found to have higher levels of sAA (Granger et al., 2007); however, in other studies, no link between low income and sAA has been found (Haushofer et al., 2011).

The present study aimed to emphasise the diversity in HPA and SAM functions in relation to temperament, cognitive abilities, language abilities and SES in children after they enter kindergarten and before they reach the age of two (in Finland, kindergartens are a part of early childhood education services for children ranging in age from one to six). The first years of life are fundamental to the calibration of stress response systems (Chiccetti \& Walker, 2001; Gunnar \& Cheatham, 2003). It is worthwhile to gather information that can be used to develop best practices to equalise the possibilities for optimal learning and wellbeing in every child.

We hypothesise that toddlers already exhibit a wide variety of abilities and behaviours when they enter kindergarten. We suppose that these differences are due to diversity in family backgrounds and the children's biological givens (gender, individual temperament, birth weight, birth height, Apgar scores). These differences might be linked to cognitive and language development. We further suppose that time spent in a kindergarten environment encumbers the children's immature stress regulative system, and that might be indicated in imbalanced HPA and SAM responses during a kindergarten day. We presume that children's individual temperaments moderate the effects of family background on stress regulation, and that some characteristics may predispose children to increased stress vulnerability. Furthermore, stress vulnerability increases the children's risks for non-optimal cognitive and language development.

This study seeks to answer the following research questions:

1. How are family background, children's biological givens, language abilities and cognitive abilities related to each other at the beginning of kindergarten in toddlers that are younger than two years of age?

2. What are the possible differences in HPA and SAM responses during a day at home and a day at kindergarten after 12 weeks (+/- four weeks) at the beginning of kindergarten?

3. Do family background and biological givens modify stress response regulation, and does stress response regulation channel children's language and cognitive development?

\section{Materials and methods}

\subsection{Participants and procedure}

The participants were recruited from 29 municipal kindergartens in metropolitan Helsinki, Finland during the years 2011-2014 as a part of the longitudinal study, "Quality of day care and the risk of social exclusion in early childhood", which was funded by the Academy of Finland. After receiving authorization from the city of Helsinki, the heads of the kindergartens were asked to participate in the study and deliver the study's consent form to parents whose children were entering the kindergartens at the beginning of next school season. The families who did not communicate in Finnish, Swedish or English were excluded, as were children with severe developmental dysfunctions. During the time of the present study, the parents of 129 children (56 girls, 73 boys) had given permission for their child to participate in this three-year follow-up study. As previously 
noted, in Finland, kindergartens are a part of early childhood education services for children ranging in age from one to six. In the present study, the mean age of the children after entering the kindergarten was 14.8 months (SD 5.2).

$<$ Table 1 about here>

The Ethics Committee on Human Studies of the University of Helsinki approved this study. We guaranteed the participants' anonymity, and the parents were aware that they were free to withdraw at any point during the study. All the study arrangements were agreed upon with the parents and the staff at the participating kindergartens. Requirements expected of the participants were made clear in the information given prior to their agreeing to participate. Participation was fully voluntary.

At the beginning of the study, a family background questionnaire and the Early Childhood Behavior Questionnaire (ECBQ) were distributed to the parents. The family background questionnaire included questions about family composition, the occurrence of learning difficulties in family members and SES. In addition, it obtained information about the participating children's birth related factors, possible current medications and developmental concerns. The child-centred information is presented in Table 1 and the family background information is presented in Table 2 .

$<$ Table 2 about here>

After the children completed a three-month (+/- one month) kindergarten adjustment period, the first saliva samples were collected and their language and cognitive abilities were evaluated. This paper presents the results of the first time point evaluations of the three-year follow-up study.

\subsection{Behavioural Assessment}

For all the participating children, the parents were asked to complete the Early Childhood Behavior Questionnaire (ECBQ), which is an extensive parent reported measure of temperament designed for children ranging in age from one to three (Putnam, Garstein, \& Rothbart, 2006). The ECBQ contains 202 items that assess the following 18 dimensions of temperament: activity level, attentional focusing, attentional shifting, cuddliness, discomfort, fear, frustration, high-intensity pleasure, impulsivity, inhibitory control, low-intensity pleasure motor activation, perceptual sensitivity, positive anticipation, sadness, shyness, sociability and soothability. The parents rated their children on each item using a 7-point Likert scale, ranging from 'extremely untrue of your child' to 'extremely true of your child'. The parents were also provided with a 'not applicable' response option to be used when the child had not been observed in the situation described. Factor analyses of the ECBQ-scales reliably recovered a three-factor solution, indicating three broad dimensions of temperament: extraversion/surgency, negative affectivity and effortful control. The ECBQ is known to be a valid and reliable instrument, and it is currently widely used in developmental research. Internal consistency (Cronbach's $\alpha$ ) in the ECBQ dimension varies from 0.93 to 0.69 (Putnam et al., 2006).

\subsection{Evaluation of Cognitive and Language Abilities}


The third edition of Bayley Scales of Infant Development (BSID III) was used to evaluate the children's cognitive and language abilities. The cognitive scale of the Bayley-III contains 91 items that assess sensorimotor development, exploration and manipulation of objects, concept formation and memory. The language scale contains 97 items that assess receptive and expressive communication. Each item is scored as either "passed" or "not passed." The evaluation is terminated when "not passed" scores have been obtained for five consecutive items. The raw scores for cognition, receptive communication and expressive communication are converted into a scaled score (a mean of 10 and a standard deviation (SD) of 3), and they are further transformed into cognitive and language composite scores (a mean of 100 and an SD of 15). The Bayley-III has acceptable levels of reliability (internal consistency $>0.86$; test-retest reliability $>0.67$ ) and concurrent validity compared to several developmental diagnostic tests for children aged 14.8-36.9 months (Bayley, 2006).

Three research assistants with a background in psychology and education served as the Bayley evaluators. The evaluators received five hours of training that consisted of observing training videotapes and observing a field assessment of three toddlers, which was administered by an experienced psychologist. The first independent evaluations were administered under the supervision of an experienced evaluator. The Bayley evaluations were conducted by appointment, at the participating kindergartens after the children had adjusted to their kindergarten routines (after a period of three months, on average).

\subsection{Salivary Cortisol and Alpha-amylase Measurements}

Five salivary cortisol and alpha-amylase samples were collected from each of the children during one day at the kindergarten and one day at home. The kindergarten teachers and the parents of the participating children were guided to collect the children's samples as follows:

- Immediately after waking;

- Thirty minutes after waking;

- One hour after waking;

- In the afternoon between 14:00 and 15:00; and

- Just before going to bed.

The teachers and parents were instructed to supervise the children so that they avoided drinking or eating for 15 minutes before the samples were collected. The parents were also asked to report their children`s medication intake and chronic illness on the saliva collection days so that it was possible to confirm that there was no use of prolonged medication that would distort the results. The timing of the collection was confirmed since the teachers and parents wrote down the time of each measurement point when they collected the children's samples. In addition, using a sampling diary the teachers and the parents were able to write down if any unusual events occurred on the sampling days.

All the salivary cortisol samples were obtained by mouthing on two-inch cotton wads (Salivette Collection Kit, Salivette, Nürmbrecth). The wet wads were placed in Salivette tubes and stored immediately in a refrigerator before being delivered to the laboratory at the Finnish Institute of Occupational Health (FIOH) in Helsinki. Salivary $\alpha$-amylase activity was analysed with a Salivary $\alpha$-Amylase Assay kit (Salimetrics). The laboratory at FIOH was also responsible for verification of the validity of the measurements.

\subsection{Statistical Analyses}


Statistical analyses were made using SPSS IBM 20 software. Descriptive statistics were obtained to illustrate the children's scores in temperament, cognitive ability and language ability. Paired sample t-tests were conducted to detect differences according to gender. Associations between the variables were investigated using Pearson's correlation coefficients (r). Linear regression analysis was conducted to detect the best predictors of the children's outcomes.

Some extreme salivary cortisol and alpha-amylase values were found. Although these values were within the normal range, they could have caused distortion in the analyses. Therefore, to avoid the violation of test assumptions caused by the skewness, all the cortisol and alpha-amylase values were investigated for outliers, which were converted equally into the most extreme values (ranging between -4 SD and +4 SD from the mean values) and measured, as recommended in Nicolson (2008).

The analyses were duplicated using the non-parametric test (Wilcoxon) to confirm that the expectation-maximization (EM) algorithm didn't distort the results. The number of children with extreme values ranged between 0-16 in every measurement point. The missing values were imputed with the EM algorithm, but only if no more than two values were missing in the series.

Descriptive statistics for the salivary cortisol and alpha-amylase measurements were obtained to demonstrate the diurnal patterns of both variables. To present the relationship between cortisol and alpha-amylase and to show them in the same figure, we logarithmically transformed the values. Moreover, to detect the variation in the biomarker variables between the kindergarten day and the home day, pairwise comparisons (t-tests) were conducted. To obtain the total diurnal response of the salivary cortisol and sAA activity, area under the curve with respect to the ground (AUCg) was calculated using the same trapezoid formula described in Pruessner, Kirschbaum, Meinlschmid, and Hellhammer (2003). Then, to demonstrate variations in the sAA activity levels after corrections for variations in the cortisol, the AUCg of the sAA activity was divided by the AUCg of the free cortisol to derive an overall ratio variable (AOC) (Ali \& Pruessner, 2012). This same ratio variable was calculated for cortisol (COA) by dividing the AUCg of the salivary cortisol by the AUCg of the sAA activity. This variable demonstrated the variations in cortisol levels when the variation in sAA activity was corrected.

In addition, the salivary cortisol awakening response (sCAR) and the salivary alpha-amylase awakening response (sAAar) were calculated (derived from the morning values between awakening, +30 minutes, and one hour after waking) as described in previous studies (identifying author, 2016).

To detect the associations between the children's biological givens, family background, cognitive and language development and stress regulation, correlation analyses (Pearson`s) were conducted.

\section{Results}

This section presents the study's results.

Relationship between family background, children's biological givens, cognitive abilities and language abilities.

Family background was slightly associated with cognitive and language abilities. The number of siblings was correlated with a child`s receptive language scores $(\mathrm{r}=-.26, \mathrm{p}=.01)$, and children with more siblings scored better in language tasks. Additionally, learning difficulties among family 
members was associated with low receptive language scores $(\mathrm{r}=-.22, \mathrm{p}=.04)$. Father`s education was positively correlated with receptive language scores $(\mathrm{r}=.22, \mathrm{p}=.04)$ and with cognitive scores $(\mathrm{r}=.21$, $\mathrm{p}=.04)$.

Furthermore, female gender was associated with higher cognitive scores $(\mathrm{r}=-.2, \mathrm{p}=.03)$, higher receptive language scores $(\mathrm{r}=-.22, \mathrm{p}=.01)$ and higher expressive language scores $(\mathrm{r}=-.19, \mathrm{p}=.04)$. In addition, there was a significant positive correlation between cognitive and receptive language scores $(\mathrm{r}=.63, \mathrm{p}<.001)$ and expressive language scores $(\mathrm{r}=.52, \mathrm{p}<.001)$. Higher Apgar scores were related to higher receptive language scores $(\mathrm{r}=.25, \mathrm{p}=.04)$.

Examination of the possible gender differences revealed that cognitive scores $[\mathrm{t}(120)=2,23$, $\mathrm{p}=.028]$, receptive language scores $[\mathrm{t}(120)=2,45, \mathrm{p}=.015]$ and expressive language scores $[\mathrm{t}(120)=2,11, \mathrm{p}=.037]$ were significantly higher in the girls (Table 3). However, no other significant gender differences were found for birth weight, height, Apgar scores (Table 1) or for temperament, negative affectivity, effortful control and surgency (Table 3).

$<$ Table 3 about here>

Furthermore, no associations between a child's temperament and cognitive and language development were found. Neither were there any correlations between other family or child background characteristics and temperament.

Due to the multiple associations with background variables and language development, linear regression analysis was conducted to detect the best predictor for the variation in the target variable. The model was constructed based on the correlation analyses with the following variables: gender, child's Apgar scores, learning difficulties in the family and number of siblings. The aim was to predict the effects of these variables on language abilities. These variables significantly predicted receptive language abilities $\left[\mathrm{F}(3,61)=3,95, \mathrm{p}=.01, \mathrm{R}^{2}=.163\right]$. However, because the effects of all the variables in the model were controlled, only gender predicted a significant variation in receptive language abilities $[\beta=-.238, \mathrm{p}=.047]$. These variables significantly predictive also expressive language $\left[\mathrm{F}(3,61)=3,18, \mathrm{p}=.03, \mathrm{R}^{2}=.135\right]$. Instead when the model were controlled, only learning difficulties in family predicted significantly variation in expressive language abilities $[\beta=-.316$, $\mathrm{p}=.015]$.

Differences in HPA and SAM responses during home day and kindergarten day after 12 weeks (+/four weeks) of the beginning of kindergarten.

On average, the children's salivary cortisol and alpha-amylase levels followed a regular diurnal pattern (see Wilcox, Granger, Szanton, \& Clark, 2014). The diurnal patterns for salivary cortisol and alpha-amylase are presented in Figure 1 and Figure 2, respectively. The salivary cortisol levels were higher in the morning; they peaked 30 minutes after awakening and then decreased slightly toward the evening with the lowest values measured just before going to bed.

In the sAA levels, there was a slight decline during the first 30 minutes of the waking hours on both days (kindergarten day and home day). After that, the values increased steadily until the afternoon, after which the values declined again towards the evening. This wave form in the profile is typical for the diurnal secretion of sAA (Wilcox et al., 2014).

$<$ Figure 1 and Figure 2 about here> 
Comparisons between the kindergarten day and home day showed that there were differences in AUCg values for cortisol [ $\mathrm{t}(105)=5,8, \mathrm{p}<.001]$. In addition, the alpha-amylase over cortisol ratio value $[\mathrm{t}(105)=-3,9, \mathrm{p}<.001]$ was higher during the home day.

\section{Possible modifiers of stress response regulation and the role of stress response regulation on children's language and cognitive abilities.}

This study also aimed to investigate the extent to which family background and a child's biological givens are associated with stress response regulation; it also aimed to examine how stress response regulation might moderate the effects of these variables on children's cognitive and language outcomes. Only modest correlations between the variables were found. Effortful control and sAA awakening response for the home day $(\mathrm{r}=.29, \mathrm{p}=.007)$ were positively correlated. The cortisol over alpha-amylase ratio value for the home day was positively associated with cognitive abilities ( $\mathrm{r}=.27$, $\mathrm{p}=.006)$. Female gender was related with the diurnal AUCg value for alpha-amylase $(\mathrm{r}=-.27$, $\mathrm{p}=.005)$ and with the cortisol awakening response $(\mathrm{r}=-.221, \mathrm{p}=.02)$, as measured on the kindergarten days. Due to the lack of a significant correlation between all the variables, further analyses were not conducted.

\section{Discussion}

The present study found that one-year-old children enter kindergarten with a wide range of biological givens, making them differentially susceptible to environmental influences. It $\mathrm{h}$ has been widely documented that family matters, and home, as a primary developmental environment, significantly affects the children's ability to either flourish or fade, developmentally. Research shows that SES is associated with a wide array of cognitive, emotional and social outcomes in children, with effects children before they are born and continues into adulthood (Sourander, 2015). A variety of mechanisms linking SES to children's wellbeing have been proposed, with most involving differences in access to material and social resources or reactions to stress-inducing conditions (Karb et al., 2012). Our study aimed to investigate the extent to which children's background characteristics are associated with children's outcomes and stress response regulation. We found that a father's high level of education is related to a child's strong cognitive abilities. This might tentatively indicate that these children could be privileged, having a more solid base for adjustment for early entry into kindergarten.

The importance of family background was further confirmed through our findings, which showed that learning difficulties in some family members predicted delayed language abilities. This might be a sign of genetic vulnerability, as has been suggested in many studies (e.g. Eischer et al., 2015; Raschle et al., 2015). On the other hand, environmental factors are known to be essential in language development (e.g. Rudolf \& Leonard, 2016; Merrit \& Klein, 2015). In our study, we found that learning difficulties in the family might indicate fewer variations in family communication, fewer enriching experiences and compromised early language development in children.

Our findings demonstrate that girls and boys differ in their language and cognitive abilities, and this should be better acknowledged in early childhood education practices. Cognitive and language abilities were stronger in the girls than the boys when children enter kindergarten. This finding implies that sociocultural traditions (including family culture) might create conditions that favour girls, as, for example, Miller and Halpern (2014) have proposed. However, some children may be more at risk than others due to weak cognitive or language development. High quality early 
education with scaffolding and sensitive professionals has the potential power to re-channel every child's development when needed, irrespective of family background or biological givens.

Several studies have documented that early age entrance to kindergartens strains the stress sensitive systems of children, resulting in possible harmful effects (e.g. Albers, Beijers, Riksen-Walraven, Sweep, \& de Weerth, 2015; Watamura et al., 2004). However, our results did not confirm the findings from previous studies, suggesting that out-of-the home early education services are compromising the wellbeing of young children. Salivary cortisol and sAA as indicators of a stress sensitive system followed, on average, a regular diurnal pattern during the kindergarten day. The asymmetrical pattern between these biomarkers has been shown to demonstrate typical secretion of cortisol and alpha-amylase (Wilcox et al., 2014).

Nevertheless, we found some differences between the home and kindergarten days. In addition, we found associations between stress responses and a child's biological characteristics. A higher alphaamylase over cortisol ratio during the home days in comparison to the kindergarten days may indicate more alerted reactivity. This is an interesting finding that requires more information about parenting styles, family activities and stressors during home days.

Interestingly, cortisol secretion was found to be more profound during the kindergarten day, which was also observed in other studies (see Vermeer \& Ijzendoorn, 2006). That might be an alarming sign (Berry et al., 2012). On the other hand, the alpha-amylase over cortisol ratio was lower during the kindergarten day, thereby indicating balanced stress response regulation with its possible positive consequences, while the opposite pattern, demonstrating higher alpha-amylase over cortisol ratio, has been linked to more blunted HPA-axis activity (Ali \& Pruessner, 2012).

In general, a study by Ali and Pruessner (2012) suggested that the alpha-amylase over cortisol ratio value is a better indicator of stress system regulation than either the cortisol over alpha-amylase ratio or alpha-amylase or cortisol alone. Regulation is needed since children certainly face multiple stressors during a kindergarten day. They have to adapt their behaviour in all kinds of relationships, both with other children and with adults. Learning challenges, minor conflicts, disappointments and frustrations commonly occur every day. Furthermore, children are forced to adjust their needs to meet the requirements of their daily routines. All these encounters might tax their emotional resources and adaptive competencies, thus activating the stress-sensitive physiological system (Watamura et al., 2004). We propose that the activated but balanced and well-regulated stress responses highlighted in our study might accelerate a child's development. The consistency and regularity of daily routines, predictive schedules and rewarding social interaction with peers and adults enhance a child's ability to face stressors.

Our research revealed noteworthy findings that are in line with previous studies, which found that temperament is related to individual differences in stress response regulation (Hill-Soderlund et al., 2015; Keller \& El-Sheikh, 2009; Fortunato et al., 2008; El-Sheikh et al., 2008). Higher morning awakening response for alpha-amylase was linked to higher effortful control, which, in extreme cases, may indicate a potential risk for anxiety (Van Veen et al., 2008). In our study, the children demonstrating high effortful control combined with elevated morning alpha-amylase may have inflexible and unadaptable mechanisms to cope accurately with challenges. Alpha-amylase has been associated with acute psychosocial stress, but as Nater and Rohleder (2009) stated, more studies are needed to examine long-term changes in alpha-amylase concentrations. In addition, meta-analysis (Schumacher, Kirschbaum, Fyd, Rich, \& Ströhle, 2013) has demonstrated that most of the studies have been conducted in laboratory or experimental settings instead of in the context of everyday life, and the participants in those studies have represented a selective sample, including for example 
psychiatric disorders such as Post Traumatic Stress Disorder (PTSD), generalised anxiety disorder or major depressive disorder.

However, we tentatively conclude that temperament might moderate development of stress response regulation. In turn, stress response regulation could channel social, cognitive and language development. By the time children enter kindergarten the differences in their stress response regulation have not yet had an influence on their cognitive and language development, as documented in our study. Therefore, adequate and sensitive co-regulation based on recognition of individual characteristics might be fundamental for supporting every child's development.

This study had some limitations that should be taken into consideration when interpreting its results. Conducting the study in a natural environment is challenging due to the limited capacity to control the accuracy of the data collection. The salivary cortisol and alpha-amylase samples were collected at the participating kindergartens and at the children's homes, and even though the teachers and parents were given proper guidelines for sample collection there was some minor variation in the sampling times. This may have an influence on the results; for example, the validity of the cortisol awakening response (as well as the alpha-amylase levels) is highly influenced by the timing of the sampling schedule. This means that an accurate time frame - beginning with the awakening timeis required to properly calculate the awakening values (Stalder et al., 2016).

This study aimed to investigate the extent to which the children's background characteristics are associated with the children's outcomes and stress response regulation. However, the study participants represent a somewhat selective sample; for example, the income level of the children's parents was higher than average. This may cause a slight distortion in the results because the variation in background characteristics was not normative. Families with a lower SES may not be willing to participate, and further studies are needed to better recruit and involve lower SES families, and to provide a more in-depth analysis of the potential differences.

Nevertheless, our study results suggest that there are differences between children due to their individual characteristics and family background when they enter kindergarten. A number of studies (Vandell \& Wolfe, 2000; Peisner-Feinberg et al., 2001; Burchinal et al., 2000) have shown that high quality early childhood education can have a positive impact on children's development and later outcomes by observing children's individual needs and intervening to address and limit developmental risk factors. High quality early childhood can be an extremely beneficial way to prevent social exclusion and guarantee social justice among children with diverse backgrounds. High quality early childhood education takes into account the fact that children vary in their sensitivity to context, and well-educated professionals can offer an enriching environment that enables all children to develop and flourish.

\section{References}

Albers, E. M., Beijers, R., Riksen-Walraven, J. M., Sweep, F. C., \& de Weerth, C. (2015) Cortisol levels of infants in center care across the first year of life: links with quality of care and infant temperament. Stress, 1-10.

Ali, N., \& Pruessner, J. C. (2012). The salivary alpha amylase over cortisol ratio as a marker to assess dysregulations of the stress systems. Physiology \& Behavior, 106, 65-72. 
Allwood, M. A., Handwerger, K., Kivlighan, K. T., Granger, D. A., \& Stroud, L. R. (2011). Direct and moderating links of salivary alpha-amylase and cortisol stress-reactivity to youth behavioral and emotional adjustment. Biological psychology, 88(1), 57-64.

Bayley, N. (2006). Bayley scales of infant and toddler development: Bayley-III. San Antonio, TX: Harcourt Assessment

Belsky, J. (2005). Differential susceptibility to rearing influences: An evolutionary hypothesis and some evidence. In B. Ellis, \& D. Bjorklund (Eds.), Origins of the social mind: Evolutionary psychology and child development (pp. 139-163). New York: Guildford.

Berntson, G.G., Cacioppo, J.T. \& Quigley, K.S. (1993). Respiratory sinus arrhythmia: Autonomic origins, physiological mechanisms, and psychophysiological implications, Psychophysiology, 30(2), 183-196.

Berry, D., Blair, C., Willoughby, M., Granger, D. A., \& Family Life Project Key Investigators. (2012). Salivary alpha-amylase and cortisol in infancy and toddlerhood: Direct and indirect relations with executive functioning and academic ability in childhood.

Psychoneuroendocrinology, 37(10), 1700-1711.

Blair, C., Granger, D., \& Peters Razza, R. (2005). Cortisol reactivity is positively related to executive function in preschool children attending Head Start. Child development, 76(3), 554-567.

Blair, C., Granger, D. A., Willoughby, M., Mills-Koonce, R., Cox, M., Greenberg, M. T., ... \& Fortunato, C. K. (2011). Salivary cortisol mediates effects of poverty and parenting on executive functions in early childhood. Child development, 82(6), 1970-1984.

Boyce, W. T., \& Ellis, B. J. (2005). Biological sensitivity to context: I. An evolutionary developmental theory of the origins and functions of stress reactivity. Development and Psychopathology, 17, 271-301.

Bradley R.H. Corwyn R.F. (2002). Socioeconomic status and child development. Annual Review of Psychology. 53, 371-99.

Burchinal, M. R., Roberts, J. E., Riggins Jr, R., Zeisel, S. A., Neebe, E., \& Bryant, D. (2000). Relating quality of center-based child care to early cognitive and language development longitudinally. Child development, 71(2), 339-357.

Chiccetti, D. \& Walker, E.F. (2001). Editorial: Stress and development:

Biological and psychological consequences. Development and Psychopathology, 13, 413-418.

Chicchetti, D. \& Rogosch, F.A. (2012). Neuroendocrine regulation and emotional adaptation in the context of child maltreatment. Monographs of the Society for Research in Child Development, 77 (2), 87-95.

Davis, E. P., \& Granger, D. A. (2009). Developmental differences in infant salivary alpha-amylase and cortisol responses to stress. Psychoneuroendocrinology, 34(6), 795-804. 
De Kloet, E.R., Fitzsimons, C.P., Datson, N.A., Meijer, O.C. \& Vreugdenhil, E. (2009). Glucocorticoid signaling and stress-related limbic susceptibility pathway: About receptors, transcription machinery and microRNA. Brain Research, 1293, 129-141.

Dougherty, L. R., Klein, D. N., Olino, T. M., Dyson, M., \& Rose, S. (2009). Increased waking salivary cortisol and depression risk in preschoolers: the role of maternal history of melancholic depression and early child temperament. Journal of Child Psychology and Psychiatry, 50(12), 1495-1503.

Dougherty, L. R., Smith, V. C., Olino, T. M., Dyson, M. W., Bufferd, S. J., Rose, S. A., \& Klein, D. N. (2013). Maternal psychopathology and early child temperament predict young children's salivary cortisol 3 years later. Journal of abnormal child psychology, 41(4), 531-542.

Dowd, J. B., Simanek, A. M., \& Aiello, A. E. (2009). Socio-economic status, cortisol and allostatic load: a review of the literature. International journal of epidemiology, dyp 277.

Eicher, J. D., Montgomery, A. M., Akshoomoff, N., Amaral, D. G., Bloss, C. S., Libiger, O., ... \& Ernst, T. (2015). Dyslexia and language impairment associated genetic markers influence cortical thickness and white matter in typically developing children. Brain imaging and behavior, 1-11.

El-Sheikh, M., Erath, S. A., Buckhalt, J. A., Granger, D. A., \& Mize, J. (2008). Cortisol and children's adjustment: The moderating role of sympathetic nervous system activity. Journal of Abnormal Child Psychology, 36(4), 601-611.

Engert, V., Vogel, S., Efanov, S. I., Duchesne, A., Corbo, V., Ali, N., \& Pruessner, J. C. (2011). Investigation into the cross-correlation of salivary cortisol and alpha-amylase responses to psychological stress. Psychoneuroendocrinology, 36(9), 1294-1302.

Evans, G.W. (2004). The Environment of Childhood Poverty. American Psychologist, 59(2), 77-92.

Evans, P. D., Fredhoi, C., Loveday, C., Hucklebridge, F., Aitchison, E., Forte, D., \& Clow, A. (2011). The diurnal cortisol cycle and cognitive performance in the healthy old. International Journal of Psychophysiology, 79(3), 371-377.

Fox, N. A., \& Reeb-Sutherland, B. C. (2010). Biological moderators of infant temperament and its relation to social withdrawal. The development of shyness and social withdrawal, 84-103.

Fortunato, C. K., Dribin, A. E., Granger, D. A., \& Buss, K. A. (2008). Salivary alpha-amylase and cortisol in toddlers: differential relations to affective behavior. Developmental psychobiology, 50(8), 807-818.

van Goozen, S. H., Matthys, W., Cohen-Kettenis, P. T., Buitelaar, J. K., \& van Engeland, H. (2000). Hypothalamic-pituitary-adrenal axis and autonomic nervous system activity in disruptive children and matched controls. Journal of the American Academy of Child \& Adolescent Psychiatry, 39(11), 1438-1445. 
Gordis, E. B., Granger, D. A., Susman, E. J., \& Trickett, P. K. (2006). Asymmetry between salivary cortisol and $\alpha$-amylase reactivity to stress: Relation to aggressive behavior in adolescents. Psychoneuroendocrinology, 31(8), 976-987.

Gunnar, M. R., Tout, K., de Haan, M., Pierce, S., \& Stanbury, K. (1997). Temperament, social competence, and adrenocortical activity in preschoolers. Developmental psychobiology, $31(1), 65-85$.

Gunnar, M. R., \& Cheatham, C. L. (2003). Brain and behavior interface: Stress and the developing brain. Infant mental health journal, 24(3), 195-211.

Gunnar, M.R., Wewerka, S., Frenn, K., Long, J.D. \& Griggs, C. (2009). Developmental changes in hypothalamus-pituitary-adrenal activity over the transition to adolescence: normative changes and associations with puberty. Dev. Psychopathol. 21, 69-85

Granger, D. A., Kivlighan, K. T., El-Sheikh, M. O. N. A., Gordis, E. B., \& Stroud, L. R. (2007). Salivary $\alpha$-amylase in biobehavioral research. Annals of the New York Academy of Sciences, 1098(1), 122-144.

Hackman, D. A., \& Farah, M. J. (2009). Socioeconomic status and the developing brain. Trends in Cognitive Sciences, 13, 65-73.

Hart, B., \& Risley, T. R. (1995). Meaningful differences in the everyday experience of young American children. Paul H Brookes Publishing.

Haushofer, J., Cornelisse, S., Joels, M., Kalenscher, T., Fehr, E., \& Haushofer, J. (2011). Low income is associated with high baseline levels and low stress reactivity of cortisol, but not alpha amylase. Working paper.

Hill-Soderlund, A. L., Holochwost, S. J., Willoughby, M. T., Granger, D. A., Gariépy, J. L., MillsKoonce, W. R., \& Cox, M. J. (2015). The developmental course of salivary alpha-amylase and cortisol from 12 to 36 months: Relations with early poverty and later behavior problems. Psychoneuroendocrinology, 52, 311-323.

Karb, R. A., Elliott, M. R., Dowd, J. B., \& Morenoff, J. D. (2012). Neighborhood-level stressors, social support, and diurnal patterns of cortisol: the Chicago Community Adult Health Study. Social science \& medicine, 75(6), 1038-1047.

Keller, P. S., \& El-Sheikh, M. (2009). Salivary alpha-amylase as a longitudinal predictor of children's externalizing symptoms: Respiratory sinus arrhythmia as a moderator of effects. Psychoneuroendocrinology, 34(5), 633-643.

Keller, P. S., El-Sheikh, M., Granger, D. A., \& Buckhalt, J. A. (2012). Interactions between salivary cortisol and alpha-amylase as predictors of children's cognitive functioning and academic performance. Physiology \& behavior, 105(4), 987-995.

Larson, K., Russ, S.A., Nelson, B.B., Olson, L.M., Halfon, N. (2015). Cognitive Ability at Kindergarten Entry and Socioeconomic Status. Pediatrics, 135, 2, 2014-0434 
McBurnett, K., Lahey, B. B., Rathouz, P. J., \& Loeber, R. (2000). Low salivary cortisol and persistent aggression in boys referred for disruptive behavior. Archives of General Psychiatry, 57(1), 38-43.

Merritt, D. H., \& Klein, S. (2015). Do early care and education services improve language development for maltreated children? Evidence from a national child welfare sample. Child abuse \& neglect, 39, 185-196.

Miller, D. I., \& Halpern, D. F. (2014). The new science of cognitive sex differences. Trends in cognitive sciences, 18(1), 37-45.

Nater, U. M., \& Rohleder, N. (2009). Salivary alpha-amylase as a non-invasive biomarker for the sympathetic nervous system: current state of research. Psychoneuroendocrinology, 34(4), 486-496

Nicolson, N.A. (2008). Measurement of cortisol. In Luecken, L \& Gallo, L.C. (eds.) Handbook of Physiological Research Methods in Health Psychology. Thousand Oaks, CA: Sage Publications, Inc. 37-73.

Obradovic, J., \& Boyce, W. T. (2009). Individual differences in behavioral, physiological, and genetic sensitivities to contexts: Implications for development and adaptation. Developmental Neuroscience, 31, 300-308.

Peisner-Feinberg, E. S., Burchinal, M. R., Clifford, R. M., Culkin, M. L., Howes, C., Kagan, S. L., \& Yazejian, N. (2001). The relation of preschool child-care quality to children's cognitive and social developmental trajectories through second grade. Child development, 72(5), $1534-1553$

Porges, S.W. (2007). The polyvagal perspective. Biological Psychology, 74:116-143.

Power, C., Li, L., \& Hertzman, C. (2008). Cognitive development and cortisol patterns in mid-life: findings from a British birth cohort. Psychoneuroendocrinology, 33(4), 530-539.

Putnam, S., Garstein, M., \& Rothbart, M. 2006. Measurement of fine-grained aspects of toddler temperament: The Early Childhood Behavior Questionnaire. Infant Behavior and Development. 29(3), 386-401

Pruessner, J. C., Kirschbaum, C., Meinlschmid, G., Hellhammer, D. H. (2003). Two formulas for the computation of the area under the curve represent measures of total hormone concentration versus time-dependent change. Psychoneuroendocrinology, 28(7), 916-931

Raschle, N. M., Becker, B. L. C., Smith, S., Fehlbaum, L. V., Wang, Y., \& Gaab, N. (2015). Investigating the Influences of Language Delay and/or Familial Risk for Dyslexia on Brain Structure in 5-Year-Olds. Cerebral Cortex, bhv267.

Rothbart, M. K. (2011). Becoming who we are: Temperament and personality in development. Guilford Press. 
Rutter, M., Thapar, A., Pine, D. S., Leckman, J. F., Scott, S., Snowling, M. J., \& Taylor, E. (2015). Resilience: concepts, findings, and clinical implications. Rutter's Child and Adolescent Psychiatry, 341-351.

Rudolph, J. M., \& Leonard, L. B. (2016). Early Language Milestones and Specific Language Impairment. Journal of Early Intervention, 38(1), 41-58.

Sapolsky, R.M., Romero, L.M. \& Munck, A.U. (2000). How Do Glucocorticoids Influence Stress Responses? Integrating Permissive, Suppressive, Stimulatory, and Preparative Actions. Endocrine Reviews 21, 55- 89.

Saridjan, N. S., Huizink, A. C., Koetsier, J. A., Jaddoe, V. W., Mackenbach, J. P., Hofman, A., ... \& Tiemeier, H. (2010). Do social disadvantage and early family adversity affect the diurnal cortisol rhythm in infants? The Generation R Study. Hormones and behavior, 57(2), 247254.

Saridjan, N. S., Henrichs, J., Schenk, J. J., Jaddoe, V. W., Hofman, A., Kirschbaum, C., ... \& Tiemeier, H. (2014). Diurnal cortisol rhythm and cognitive functioning in toddlers: the Generation R Study. Child Neuropsychology, 20(2), 210-229.

Sattler, J. M. (1988). Assessment of children (3rd ed.). San Diego, CA: Jerome M. Sattler.

Schumacher, S., Kirschbaum, C., Fydrich, T., \& Ströhle, A. (2013). Is salivary alpha-amylase an indicator of autonomic nervous system dysregulations in mental disorders?-A review of preliminary findings and the interactions with cortisol. Psychoneuroendocrinology, 38(6), 729-743.

Shirtcliff, E. A., Granger, D. A., Booth, A., \& Johnson, D. (2005). Low salivary cortisol levels and externalizing behavior problems in youth. Development and psychopathology, 17(01), 167184.

Siegel, D. J. (2015). Interpersonal neurobiology as a lens into the development of wellbeing and reslience. Children Australia, 40(02), 160-164.

Sourander, A. (2015). Challenges in children's enrolment to psychosocial services. European child \& adolescent psychiatry, 24(4), 361-363.

Stalder, T., Kirschbaum, C., Kudielka, B. M., Adam, E. K., Pruessner, J. C., Wüst, S., ... \& Miller, R. (2016). Assessment of the cortisol awakening response: Expert consensus guidelines. Psychoneuroendocrinology, 63, 414-432.

Vandell, D., \& Wolfe, B. (2000). Child care quality: Does it matter and does it need to be improved? (Vol. 78). University of Wisconsin--Madison, Institute for Research on Poverty. 
Van Veen, J. F., Van Vliet, I. M., DeRijk, R. H., Van Pelt, J., Mertens, B., \& Zitman, F. G. (2008). Elevated alpha-amylase but not cortisol in generalized social anxiety disorder. Psychoneuroendocrinology, 33(10), 1313-1321.

Vermeer, H. J., \& van IJzendoorn, M. H. (2006). Children's elevated cortisol levels at daycare: A review and meta-analysis. Early Childhood Research Quarterly, 21(3), 390-401.

Vernon-Feagans, L., Garrett-Peters, P., Willoughby, M., Mills-Koonce, R., \& Family Life Project Key Investigators. (2012). Chaos, poverty, and parenting: Predictors of early language development. Early Childhood Research Quarterly, 27(3), 339-351.

Watamura, S. E., Donzella, B., Kertes, D. A., \& Gunnar, M. R. (2004). Developmental changes in baseline cortisol activity in early childhood: Relations with napping and effortful control. Developmental Psychobiology, 45(3), 125-133.

Wilcox, R. R, Granger, D. A., Szanton, S., \& Clark, F. (2014). Diurnal patterns and associations among salivary cortisol, DHEA and alpha-amylase in older adults. Physiology and Behavior, 129, 11-16. 
Table 1. Children's birth characteristics

\begin{tabular}{|c|c|c|c|c|}
\hline & & $\mathrm{N}$ & Mean & $\mathrm{Sd}$ \\
\hline \multicolumn{5}{|c|}{ Birth height $(\mathrm{cm})$} \\
\hline & Girls & 37 & 49,46 & 2,77 \\
\hline & Boys & 49 & 49,14 & 7,06 \\
\hline & total & 86 & 49,3 & 4,92 \\
\hline \multicolumn{5}{|c|}{ Birth weight (kg) } \\
\hline & Girls & 37 & 3,42 & 0,62 \\
\hline & Boys & 48 & 3,56 & 0,59 \\
\hline & total & 85 & 3,49 & $\mathbf{0 , 6 1}$ \\
\hline \multirow[t]{3}{*}{ Apgar (1-10) } & Girls & 30 & 8,87 & 0,78 \\
\hline & Boys & 39 & 8,87 & 0,08 \\
\hline & total & 69 & 8,87 & 0,43 \\
\hline
\end{tabular}


Table 2. Family background characteristics.

\begin{tabular}{|c|c|c|}
\hline \multicolumn{2}{|c|}{ Family income per year (euros) } & $\begin{array}{l}\text { Families } \\
(\%)\end{array}$ \\
\hline & $<200000$ & 5,1 \\
\hline & $20000-35000$ & 11,2 \\
\hline & $35000-50000$ & 9,2 \\
\hline & $50000-65000$ & 21,4 \\
\hline & $65000-80000$ & 23,5 \\
\hline & $>800000$ & 29,6 \\
\hline \multicolumn{2}{|c|}{ Number of children in families } & $\begin{array}{l}\text { Families } \\
(\%)\end{array}$ \\
\hline & 1 & 49,5 \\
\hline & 2 & 29,7 \\
\hline & 3 & 17,8 \\
\hline & $>3$ & 3 \\
\hline Parents educational level & Mother $(\%)$ & Father $(\%)$ \\
\hline \multicolumn{3}{|l|}{ basic education } \\
\hline high school & 23,5 & 27,3 \\
\hline graduate & 76,5 & 72,7 \\
\hline \multicolumn{3}{|l|}{ schooling } \\
\hline none & 2,9 & 15,2 \\
\hline vocational & 17,6 & 15,2 \\
\hline college & 31,4 & 30,3 \\
\hline university & 48,1 & 39,3 \\
\hline
\end{tabular}


Table 3. Children's cognitive and language abilities and temperament

\begin{tabular}{crrr}
\hline & N & Mean & Sd \\
\hline $\begin{array}{c}\text { Cognitive abilities } \\
\text { girls }\end{array}$ & 54 & 12,17 & 3,64 \\
boys & 68 & 10,68 & 3,69 \\
total & $\mathbf{1 2 2}$ & $\mathbf{1 1 , 3 4}$ & $\mathbf{3 , 7 3}$ \\
\hline Receptive language abilities & & & \\
girls & 54 & 10,88 & 3,83 \\
boys & 68 & 9,22 & 3,61 \\
total & $\mathbf{1 2 2}$ & $\mathbf{1 0 , 7 4}$ & $\mathbf{3 , 6 3}$ \\
\hline Expressive language abilities & & & \\
girls & 54 & 11,63 & 3,61 \\
boys & 68 & 10,03 & 3,52 \\
total & $\mathbf{1 2 2}$ & $\mathbf{1 0 , 7 4}$ & $\mathbf{3 , 6 3}$ \\
\hline Negative affectivity & & & \\
girls & 42 & 3,04 & 0,75 \\
boys & 59 & 2,89 & 0,81 \\
total & $\mathbf{1 0 1}$ & $\mathbf{2 , 9 7}$ & $\mathbf{0 , 7 8}$ \\
\hline Effortful control & & & \\
girls & 42 & 4,51 & 0,73 \\
boys & 59 & 4,35 & 0,93 \\
total & $\mathbf{1 0 1}$ & $\mathbf{4 , 4 3}$ & $\mathbf{0 , 8 3}$ \\
\hline Surgency extraversion & & & \\
girls & 42 & 4,75 & 0,86 \\
boys & 59 & 4,59 & 0,99 \\
total & $\mathbf{1 0 1}$ & $\mathbf{4 , 6 7}$ & $\mathbf{0 , 9 1}$ \\
\hline & & &
\end{tabular}


Figure 1. Salivary cortisol and alpha-amylase during kindergarten day (A)

Figure 2. Salivary cortisol and alpha-amylase during home day (B) 